\title{
Modules over axial algebras
}

\author{
Tom De Medts* \\ Michiel Van Couwenberghe ${ }^{\dagger}$
}

December 6, 2018

\begin{abstract}
We introduce axial representations and modules over axial algebras as new tools to study axial algebras. All known interesting examples of axial algebras fall into this setting, in particular the Griess algebra whose automorphism group is the Monster group. Our results become especially interesting for Matsuo algebras. We vitalize the connection between Matsuo algebras and 3-transposition groups by relating modules over Matsuo algebras with representations of 3-transposition groups. As a by-product, we define, given a Fischer space, a group that can fulfill the role of a universal 3-transposition group.
\end{abstract}

Keywords. axial algebras, modules, axial representations, Matsuo algebras, 3-transposition groups, Fischer spaces.

MSC2010. primary: 17A99, 20B25, 20F29, 20C05; secondary: 17B69, 20C34.

\section{Introduction}

In 1982, Robert Griess constructed a commutative non-associative algebra, called the Griess algebra, in order to prove the existence of the Monster group [Gri82]. Igor Frenkel, James Lepowsky and Arne Meurman observed that (a deformation of) this Griess algebra can be retrieved as a weight-2 component of a vertex operator algebra (VOA) $V^{\natural}$ [FLM88]. Richards Borcherds then obtained a proof of the moonshine conjectures by studying this VOA [Bor86].

The weight-2 components of VOAs similar to $V^{\natural}$ give rise to algebras with properties like the ones for the Griess algebra. Alexander Ivanov defined Majorana algebras in an attempt to axiomatize such algebras. The definition of an axial algebra was introduced by Jonathan Hall, Sergey Shpectorov and Felix Rehren in 2015 by stripping away some of the requirements in the definition of Majorana algebras in such a way that many results concerning Majorana algebras still remain true.

\footnotetext{
*tom.demedts@ugent.be

${ }^{\dagger}$ michiel.vancouwenberghe@ugent.be [PhD Fellow of the Research Foundation - Flanders (Belgium) (F.W.O.-Vlaanderen)] (corresponding author)
} 
Since axial algebras have only recently been defined, this new research subject is very much unexplored and it is not even clear whether the definition of an axial algebra is yet in its final stage.

In this paper, we provide some new tools to study axial algebras. First, we define axial representations of a group as a generalization of Majorana representations which were introduced in [IPS10]. This notion is perhaps more foundational than the definition of an axial algebra itself, since it describes the connection between axial algebras and groups, very much like the connection between the Griess algebra and the Monster group.

Second, we present a natural definition of modules over axial algebras. If an axial algebra $A$ is an axial representation of a group $G$, each $A$-module gives rise to a $\mathrm{U}(A)$-module where $\mathrm{U}(A)$ is a certain central extension of $G$; see Theorem 3.9 below. In this sense, our definition of modules over axial algebras reinforces the connection between axial algebras and groups.

Third, we investigate the theory of modules more thoroughly for Matsuo algebras over Fischer spaces, an important class of examples of axial algebras. In this case, the group $\mathrm{U}(A)$ will be a universal 3-transposition group related to the given Fischer space, as we explain in Theorem 4.13. (The well known connection between 3-transposition groups and Fischer spaces is due to Francis Buekenhout [Bue74].) This enables us to construct a module for the Matsuo algebra out of every $\mathrm{U}(A)$-module; see Theorem 5.1 . This correspondence between modules over Matsuo algebras and $\mathrm{U}(A)$-modules is not one-to-one; it turns out that 1-eigenvectors in the modules over the Matsuo algebra play a special role (see Corollary 5.3) and almost always indicate the presence of a regular module as submodule, and this is the content of Theorems 5.8 and 5.11 .

Acknowledgment. We have benefited from fruitful discussions between Jonathan Hall and the second author during a visit at Michigan State University. We thank the two anonymous referees of earlier versions of this paper for their suggestions, which improved the exposition of the results at many places.

\section{Axial algebras}

This section provides an introduction into the realm of axial algebras. We use the definition by J. Hall, F. Rehren and S. Shpectorov in [HRS15b]. This definition of an axial algebra resembles a property satisfied by idempotents in associative and Jordan algebras, namely the existence of a Peirce decomposition. The right multiplication operator of an idempotent in a Jordan algebra is diagonalizable and the decomposition into eigenvectors is compatible with the multiplication, in the sense that the multiplication of eigenvectors is described by a fusion rule.

Definition 2.1. Let $R$ be a commutative ring with identity. A fusion rule is a pair $(\Phi, \star)$ such that $\Phi \subseteq R$ and $\star: \Phi \times \Phi \rightarrow 2^{\Phi}$ is a symmetric map.

Definition 2.2. Let $R$ be a commutative ring with identity, $A$ a commutative (not necessarily associative) $R$-algebra and $e \in A$ an idempotent. For each $\phi \in R$ we denote 


\begin{tabular}{c|ccc}
$\star$ & 1 & 0 & $\alpha$ \\
\hline 1 & $\{1\}$ & $\emptyset$ & $\{\alpha\}$ \\
0 & $\emptyset$ & $\{0\}$ & $\{\alpha\}$ \\
$\alpha$ & $\{\alpha\}$ & $\{\alpha\}$ & $\{1,0\}$
\end{tabular}

Table 1: the Jordan fusion rule $\Phi(\alpha)$

the eigenspace of $e$ with eigenvalue $\phi$ by

$$
A_{\phi}^{e}=\{a \in A \mid a e=\phi a\} .
$$

For each subset $\Lambda \subseteq R$, let

$$
A_{\Lambda}^{e}=\bigoplus_{\phi \in \Lambda} A_{\phi}^{e},
$$

with the convention that $A_{\emptyset}^{e}=\{0\}$. We call $e$ a $(\Phi, \star)$-diagonalizable idempotent (or an axis) for the fusion rule $(\Phi, \star)$ if

$$
A=\bigoplus_{\phi \in \Phi} A_{\phi}^{e}
$$

and

$$
A_{\phi}^{e} \cdot A_{\psi}^{e} \subseteq A_{\phi \star \psi}^{e}
$$

for all $\phi, \psi \in \Phi$. This means that the product of a $\phi$-eigenvector and a $\psi$-eigenvector is a sum of $\chi$-eigenvectors where $\chi$ runs through $\phi \star \psi$.

Definition 2.3. A $(\Phi, \star)$-axial algebra is a pair $(A, \Omega)$ where:

(i) $A$ is a commutative (not necessarily associative) $R$-algebra and,

(ii) $\Omega \subset A$ is a generating set of $(\Phi, \star)$-axes for $A$.

We will often omit the set $\Omega$ in our notation.

Example 2.4. Idempotents of Jordan algebras are $\Phi(\alpha)$-diagonalizable idempotents for $\alpha=\frac{1}{2}$ where $\Phi(\alpha)$ denotes the Jordan fusion rule described by Table 1 [Jac68, p. 119].

The important connection between some axial algebras and groups arises from the special case of a $\mathbb{Z} / 2 \mathbb{Z}$-graded fusion rule.

Definition 2.5. (i) A fusion rule $(\Phi, \star)$ is called $\mathbb{Z} / 2 \mathbb{Z}$-graded if $\Phi$ can be partitioned into two subsets $\Phi_{+}$and $\Phi_{-}$such that

$$
\begin{aligned}
& \phi \star \psi \subseteq \Phi_{+} \text {whenever } \phi, \psi \in \Phi_{+}, \\
& \phi \star \psi \subseteq \Phi_{+} \text {whenever } \phi, \psi \in \Phi_{-}, \\
& \phi \star \psi \subseteq \Phi_{-} \text {whenever } \phi \in \Phi_{+} \text {and } \psi \in \Phi_{-} .
\end{aligned}
$$




\begin{tabular}{c|cccc}
$\star$ & 1 & 0 & $\frac{1}{4}$ & $\frac{1}{32}$ \\
\hline 1 & 1 & $\emptyset$ & $\frac{1}{4}$ & $\frac{1}{32}$ \\
0 & $\emptyset$ & 0 & $\frac{1}{4}$ & $\frac{1}{32}$ \\
$\frac{1}{4}$ & $\frac{1}{4}$ & $\frac{1}{4}$ & 1,0 & $\frac{1}{32}$ \\
$\frac{1}{32}$ & $\frac{1}{32}$ & $\frac{1}{32}$ & $\frac{1}{32}$ & $1,0, \frac{1}{4}$
\end{tabular}

Table 2: fusion rule of the Griess algebra

(ii) Let $(A, \Omega)$ be a $(\Phi, \star)$-axial algebra for some $\mathbb{Z} / 2 \mathbb{Z}$-graded fusion rule $(\Phi, \star)$. We associate to each $(\Phi, \star)$-axis $e$ of $A$ a Miyamoto involution $\tau_{e} \in \operatorname{Aut}(A)$ defined by linearly extending

$$
a^{\tau_{e}}=\left\{\begin{array}{rl}
a & \text { if } a \in A_{\Phi_{+}}^{e} \\
-a & \text { if } a \in A_{\Phi_{-}}^{e}
\end{array} .\right.
$$

Because of the $\mathbb{Z} / 2 \mathbb{Z}$-grading of the fusion rule, these maps define automorphisms of $A$. Note that, at this point, we allow $A_{\Phi_{-}}^{e}$ to be trivial and hence $\tau_{e}$ to be trivial. However, when $A_{\Phi_{-}}^{e} \neq 0$, these automorphisms are indeed involutions.

(iii) We call the subgroup $\left\langle\tau_{e} \mid e \in \Omega\right\rangle \leq \operatorname{Aut}(A)$ the Miyamoto group of the axial algebra $(A, \Omega)$, and we denote it by $\operatorname{Miy}(A, \Omega)$.

(iv) We say that $\Omega \subset A$ is Miyamoto-closed when it is invariant under $\operatorname{Miy}(A, \Omega)$.

Example 2.6. (i) The Jordan fusion rule $\Phi(\alpha)$ from Table 1 is $\mathbb{Z} / 2 \mathbb{Z}$-graded with $\Phi_{+}=\{1,0\}$ and $\Phi_{-}=\{\alpha\}$.

(ii) The Griess algebra is a 196884-dimensional real axial algebra that satisfies the fusion rule from Table 2 [Iva09, Lemma 8.5.1, p. 209]. This fusion rule is $\mathbb{Z} / 2 \mathbb{Z}$-graded with $\Phi_{+}=\left\{1,0, \frac{1}{4}\right\}$ and $\Phi_{-}=\left\{\frac{1}{32}\right\}$. The Miyamoto involutions of the generating set of axes of the Griess algebra are called Majorana involutions. They generate the full automorphism group of the Griess algebra which is better known as the Monster group; see [Iva09, Proposition 8.6.2, p. 210] and [Tit84, p. 497].

Definition 2.7. Let $k$ be a field. A Frobenius axial algebra is an axial $k$-algebra equipped with a bilinear form

$$
\langle\cdot, \cdot\rangle: A \times A \rightarrow k
$$

such that $\langle x a, b\rangle=\langle a, x b\rangle$ for all $a, b, x \in A$. We call this bilinear form the Frobenius form.

It is easy to verify that if $A$ is a Frobenius axial algebra $A$, then for each axis $a \in A$, the eigenspaces $A_{\phi}^{a}$ and $A_{\psi}^{a}$ are perpendicular for distinct $\phi$ and $\psi$ [HRS15a, Proposition 3].

It is already clear from Example 2.6(ii) that there exists an important connection between axial algebras and groups. This connection comes from the following situation which is a generalization of a Majorana representation defined in [IPS10. 
Definition 2.8. Let $G$ be a group generated by a set $D$ of elements of order at most 2 . An axial representation of $(G, D)$ is an isomorphism $\xi: G \rightarrow \operatorname{Miy}(A, \Omega)$, where

(i) $(A, \Omega)$ is a $(\Phi, \star)$-axial algebra for a $\mathbb{Z} / 2 \mathbb{Z}$-graded fusion rule $(\Phi, \star)$,

(ii) $\Omega$ is Miyamoto-closed,

(iii) $\xi(D)=\left\{\tau_{e} \in \operatorname{Aut}(A) \mid e \in \Omega\right\}$.

We will often say that $(A, \Omega)$ is an axial representation of $(G, D)$ and we will simply identify $G$ with $\operatorname{Miy}(A, \Omega)$ and $D$ with the set $\left\{\tau_{e} \mid e \in \Omega\right\}$.

Proposition 2.9 ([eh15, Lemma 2.4.1]). Let $(A, \Omega)$ be an axial representation of $(G, D)$. For each $t \in \operatorname{Aut}(A)$ and each $(\Phi, \star)$-axis $a \in A, a^{t}$ is again a $(\Phi, \star)$-axis and $\left(\tau_{a}\right)^{t}=\tau_{a^{t}}$. In particular, $\left(\tau_{x}\right)^{\tau_{y}}=\tau_{x^{\tau_{y}}}$ for all $x, y \in \Omega$.

Remark 2.10. (i) Of course, every axial algebra $(A, \Omega)$ with a $\mathbb{Z} / 2 \mathbb{Z}$-graded fusion rule such that $\Omega$ is Miyamoto-closed, is an axial representation for some $(G, D)$, namely $G=\operatorname{Miy}(A, \Omega)$ and $D=\left\{\tau_{e} \mid e \in \Omega\right\}$. The more interesting question is: which groups $G$ admit an axial representation (for some generating set $D$ of elements of order at most 2)?

(ii) We emphasize that in Definition 2.8 , we allow the possibility that $D$ contains the trivial element, or equivalently, that $\tau_{e}$ is trivial for some $e \in \Omega$.

(iii) Let $G$ be a group generated by a set $D$ of elements of order at most 2 which is invariant under conjugation. If $\xi: G \rightarrow \operatorname{Miy}(A, \Omega)$ is an isomorphism satisfying (i) and (iii) of Definition 2.8, then $\Omega$ is not necessarily Miyamoto-closed, but we can always replace $\Omega$ by the possibly larger set of axes

$$
\Omega^{\prime}:=\left\{e \in A \text { an axis } \mid \tau_{e}=\tau_{f} \text { for some } f \in \Omega\right\}
$$

without changing the Miyamoto group. It now follows from Proposition 2.9 that $\Omega^{\prime}$ is indeed Miyamoto-closed, and hence $\left(A, \Omega^{\prime}\right)$ is an axial representation of $(G, D)$.

If $(A, \Omega)$ is an axial representation for $(G, D)$, then the map $\tau: \Omega \rightarrow D: e \mapsto \tau_{e}$ is not necessarily a bijection. This motivates the following definition, which we have taken from [HSS17, Definition 6.8].

Definition 2.11. Let $(A, \Omega)$ be an axial representation of $(G, D)$. We call the axial representation of unique type if the map $\tau: \Omega \rightarrow D: e \mapsto \tau_{e}$ is a bijection.

According to [HSS17, Theorem 6.10], "most" axial representations that satisfy the Jordan fusion rule are of unique type; in particular, those arising from 3-transposition groups fall in this class (see Proposition 4.10(iii) below). Axial representations of unique type behave more nicely than others; see, for instance, Proposition 3.7 below.

Example 2.12. Not every axial representation is of unique type. Indeed, consider the 3 -dimensional algebra spanned by vectors $\mathbb{1}, u$ and $v$ and commutative product defined by $u^{2}=v^{2}=\mathbb{1}, u v=0$ and such that $\mathbb{1}$ is the identity. This is a Jordan algebra of Clifford type generated by the idempotents $e_{1}=\frac{1}{2}+\frac{1}{2} u, e_{2}=\frac{1}{2}+\frac{1}{2} v, \mathbb{1}-e_{1}$ and $\mathbb{1}-e_{2}$. For the $\mathbb{Z} / 2 \mathbb{Z}$-grading of the Jordan fusion rule $\Phi\left(\frac{1}{2}\right)$ we get $\tau_{e_{1}}=\tau_{\mathbb{1}-e_{1}}$ and $\tau_{e_{2}}=\tau_{\mathbb{1}-e_{2}}$. This leads to an axial representation of $(\mathbb{Z} / 2 \mathbb{Z})^{2}$, which is not of unique type. 


\section{Modules over axial algebras}

The definition of an axial algebra leads to the following natural definition for modules over axial algebras, our main object of interest.

Definition 3.1. Let $R$ be a commutative ring with identity and $(A, \Omega)$ a $(\Phi, \star)$-axial $R$-algebra. Let $M$ be an $R$-module equipped with a (right) $R$-bilinear action of $A$,

$$
M \times A \rightarrow M:(m, a) \mapsto m \cdot a .
$$

For each $e \in A$ and each $\phi \in R$, let $M_{\phi}^{e}=\{m \in M \mid m \cdot e=\phi m\}$. Define, as usual, for every nonempty $\Lambda \subset R, M_{\Lambda}^{e}=\bigoplus_{\phi \in \Lambda} M_{\phi}^{e}$ and $M_{\emptyset}^{e}=\{0\}$. We call $M$ an $A$-module if, for each $e \in \Omega$,

(i) there exists a decomposition $M=\bigoplus_{\phi \in \Phi} M_{\phi}^{e}$ and

(ii) $m \cdot a \in M_{\phi \star \psi}^{e}$ whenever $m \in M_{\phi}^{e}$ and $a \in A_{\psi}^{e}$.

The collection of all $A$-modules forms a category $\mathbf{M o d}_{A}$; an $R$-module morphism $\theta$ between two $A$-modules $M_{1}, M_{2}$ is an A-module morphism if $\theta(m \cdot a)=\theta(m) \cdot a$ for all $m \in M$ and all $a \in A$.

Observe that $A$ is itself an $A$-module; we refer to it as the regular module for $A$.

We can extend the definition of Miyamoto involutions and Frobenius forms to arbitrary modules over axial algebras.

Definition 3.2. Let $A$ be an axial algebra for a $\mathbb{Z} / 2 \mathbb{Z}$-graded fusion rule and $M$ an $A$-module. For every $(\Phi, \star)$-axis $e \in A$ we define the Miyamoto involution $\mu_{e} \in \operatorname{GL}(M)$ of $M$ :

$$
m^{\mu_{e}}=\left\{\begin{aligned}
m & \text { if } m \in M_{\Phi_{+}}^{e} \\
-m & \text { if } m \in M_{\Phi_{-}}^{e} .
\end{aligned}\right.
$$

Notice that $\mu_{e}$ is possibly trivial (namely when $M_{\Phi_{-}}^{e}=0$ ).

Definition 3.3. Let $A$ be an axial $k$-algebra for a field $k$. A Frobenius $A$-module is an $A$-module $M$ equipped with a bilinear form, called the Frobenius form,

$$
\langle\cdot, \cdot\rangle: M \times M \rightarrow k
$$

such that $\langle m \cdot a, n\rangle=\langle m, n \cdot a\rangle$ for all $m, n \in M$ and $a \in A$.

The following proposition is reminiscent of Maschke's theorem for linear representations of finite groups.

Proposition 3.4. Let $A$ be a finite-dimensional $k$-algebra and $M$ a Frobenius A-module. Let $N$ be an $A$-submodule of $M$, i.e. a k-subspace for which $N \cdot A \subseteq N$, such that the Frobenius form is non-degenerate on $N$. Then there exists an $A$-submodule $N_{0}$ of $M$ such that $M=N \oplus N_{0}$. 
Proof. Let $N_{0}=\{m \in M \mid\langle m, n\rangle=0$ for all $n \in N\}$. For all $a \in A, n_{0} \in N_{0}$ and $n \in N$, we have $\left\langle n_{0} \cdot a, n\right\rangle=\left\langle n_{0}, n \cdot a\right\rangle=0$ since $n \cdot a \in N$. Hence $n_{0} \cdot a \in N_{0}$ and $N_{0}$ is an $A$-submodule. Since we require the Frobenius form to be non-degenerate on $N$, it follows from the properties of orthogonal complements in finite-dimensional vector spaces that $M=N \oplus N_{0}$.

In the remainder of this section, we will study modules over axial representations. In Theorem 3.9 below, we will show that every module over an axial representation $(A, \Omega)$ of $(G, D)$ leads to a group representation of a central extension $\mathrm{U}(A, \Omega)$ of $G$. We start by presenting the definition of this central extension $\mathrm{U}(A, \Omega)$ and relating it to $G$.

Definition 3.5. Let $(A, \Omega)$ be an axial representation of $(G, D)$. We define the group $\mathrm{U}(A, \Omega)$ as the group with presentation

$$
\left\langle\begin{array}{l|l}
t_{e} \text { for each } e \in \Omega \mid \begin{array}{l}
\left(t_{e}\right)^{2}=1 \\
\left(t_{x}\right)^{t_{y}}=t_{x^{\tau} y}
\end{array} \text { for all } e \in \Omega, y \in \Omega
\end{array}\right\rangle .
$$

Note that we need $\Omega$ to be Miyamoto-closed in order to define this group.

We prove some useful properties of this group.

Proposition 3.6. Let $(A, \Omega)$ be an axial representation of $(G, D)$.

(i) The map $\tau: \mathrm{U}(A, \Omega) \rightarrow G: t_{e} \mapsto \tau_{e}$ is a group epimorphism.

(ii) The group $\mathrm{U}(A, \Omega)$ is a central extension of $G$.

(iii) If $\Omega$ is finite, then so is $\mathrm{U}(A, \Omega)$.

Proof. (i) Consider the map $\tau: \mathrm{U}(A, \Omega) \rightarrow G$ defined by $t_{e} \mapsto \tau_{e}$ for all $e \in \Omega$. This map is a group homomorphism since all relations that define $\mathrm{U}(A, \Omega)$ hold in $G$ by Proposition 2.9. Since the elements $\tau_{e}$ generate $G$, this map is surjective.

(ii) We prove that the kernel of $\tau$ is contained in the center of $\mathrm{U}(A, \Omega)$. Let $u:=$ $t_{x_{1}} t_{x_{2}} \cdots t_{x_{n}} \in \operatorname{ker}(\tau)$ where $x_{1}, x_{2}, \ldots, x_{n} \in \Omega$. Then $\tau_{x_{1}} \tau_{x_{2}} \cdots \tau_{x_{n}}=1$ and thus, for all $e \in \Omega$,

$$
t_{e}^{u}=t_{e}^{t_{x_{1}} t_{x_{2}} \cdots t_{x_{n}}}=t_{e^{\tau_{x_{1}} \tau_{x_{2}} \cdots \tau_{x_{n}}}}=t_{e} .
$$

Thus $u$ commutes with all $t_{e}$ and is therefore contained in the center of $\mathrm{U}(A, \Omega)$.

(iii) We will show that every element of $\mathrm{U}(A, \Omega)$ can be written as a product of at most $|\Omega|$ elements $t_{e}$. Since only finitely many such products exist, $\mathrm{U}(A, \Omega)$ must be finite.

Consider a product of more than $|\Omega|$ elements $t_{e}$. Some element $t_{x}$ will then appear at least twice and we can use the relation

$$
t_{x} t_{e_{1}} t_{e_{2}} \cdots t_{e_{n}} t_{x}=\left(t_{e_{1}} t_{e_{2}} \cdots t_{e_{n}}\right)^{t_{x}}=t_{\left(e_{1}\right)^{\tau_{x}}} t_{\left(e_{2}\right)^{\tau_{x}}} \cdots t_{\left(e_{n}\right)^{\tau_{x}}}
$$

to rewrite this element as a product of fewer $t_{e}$ 's. 
For axial representations of unique type, we can say even more.

Proposition 3.7. Suppose $(A, \Omega)$ is an axial representation of $(G, D)$ of unique type. Then the following hold.

(i) $G$ is centerless.

(ii) $\mathrm{U}(A, \Omega)$ only depends on $G$ and $D$.

(iii) The kernel of the group homomorphism $\tau: \mathrm{U}(A, \Omega) \rightarrow G: t_{e} \mapsto \tau_{e}$ coincides with the center of $\mathrm{U}(A, \Omega)$.

Proof. (i) Suppose $\tau_{x_{1}} \tau_{x_{2}} \cdots \tau_{x_{n}} \in \mathrm{Z}(G)$. Then, for all $e \in \Omega$,

$$
\tau_{e}=\left(\tau_{e}\right)^{\tau_{x_{1}} \tau_{x_{2}} \cdots \tau_{x_{n}}}=\tau_{e^{\tau_{x_{1}}} \tau_{x_{2}} \cdots \tau_{x_{n}}}
$$

Since $(A, \Omega)$ is of unique type, the elements of $\mathrm{Z}(G)$ act trivially on $\Omega$ and hence also on $A$.

(ii) The presentation of Definition 3.5 defining $\mathrm{U}(A, \Omega)$ can be retrieved from $(G, D)$. Since $(A, \Omega)$ is of unique type, the generators can be identified with the set $D$ and the relations are the conjugacy relations between elements of $D$.

(iii) This follows from part (i) and Proposition 3.6(ii),

Remark 3.8. It is possible and might seem more natural to define a group $\mathrm{U}(G, D)$ in a similar way as in Definition 3.5 using the elements of $D$ rather than the idempotents of an axial representation. This group $\mathrm{U}(G, D)$ was already studied by H. Cuypers and J. Hall in the context of 3 -transposition groups [Hal06, Proposition 2.1]. If $(A, \Omega)$ is an axial representation of $(G, D)$ of unique type, then $\mathrm{U}(G, D) \cong \mathrm{U}(A, \Omega)$. However, for the axial representation of $(\mathbb{Z} / 2 \mathbb{Z})^{2}$ from Example 2.12, which is not of unique type, we have $\mathrm{U}(G, D) \cong(\mathbb{Z} / 2 \mathbb{Z})^{2}$ while $\mathrm{U}(A, \Omega) \cong(\mathbb{Z} / 2 \mathbb{Z})^{4}$.

We are now able to state one of our main theorems.

Theorem 3.9. Let $(A, \Omega)$ be an axial representation of $(G, D)$ and let $M$ be an $A$-module. The map defined by

$$
\mu: \mathrm{U}(A, \Omega) \rightarrow \mathrm{GL}(M): t_{e} \mapsto \mu_{e}
$$

for all $e \in \Omega$, is a group homomorphism.

We start by proving the following lemma, the proof of which is inspired by Reh15, Lemma 2.4.1].

Lemma 3.10. For all $m \in M, a \in A$ and all $x, y \in \Omega$ we have:

(i) $(m \cdot a)^{\mu_{x}}=m^{\mu_{x}} \cdot a^{\tau_{x}}$,

(ii) $\left(M_{\phi}^{y}\right)^{\mu_{x}}=M_{\phi}^{y^{\tau_{x}}}$,

(iii) $\left(\mu_{x}\right)^{\mu_{y}}=\mu_{x} \tau_{y}$. 
Proof. (i) Let $m \in M_{\Phi_{+}}^{x}$ and $a \in A_{\Phi_{+}}^{x}$, then $m \cdot a \in M_{\Phi_{+}}^{x}$ and therefore $(m \cdot a)^{\mu_{x}}=m \cdot a$, $m^{\mu_{x}}=m$ and $a^{\tau_{x}}=a$. For $m \in M_{\Phi_{-}}^{x}$ and $a \in A_{\Phi_{+}}^{x}$ (resp. $m \in M_{\Phi_{+}}^{x}$ and $\left.a \in A_{\Phi_{-}}^{x}\right)$ we have $m \cdot a \in M_{\Phi_{-}}$and hence $(m \cdot a)^{\mu_{x}}=-m \cdot a, m^{\mu_{x}}=-m$ (resp. $m^{\mu_{x}}=m$ ) and $a^{\tau_{x}}=a\left(\right.$ resp. $\left.a^{\tau_{x}}=-a\right)$. Finally, let $m \in M_{\Phi_{-}}^{x}$ and $a \in A_{\Phi_{-}}^{x}$. Now $m \cdot a \in M_{\Phi_{+}}^{x}$ and thus $(m \cdot a)^{\mu_{x}}=m \cdot a, m^{\mu_{x}}=-m$ and $a^{\tau_{x}}=-a$. Since $M=M_{\Phi_{+}}^{x} \oplus M_{\Phi_{-}}^{x}$ and $A=A_{\Phi_{+}}^{x} \oplus A_{\Phi_{-}}^{x}$, the desired property follows by linearity of the action of $A$ on $M$.

(ii) Let $m \in M_{\phi}^{y}$. By (i), it follows that $m^{\mu_{x}} \cdot y^{\tau_{x}}=(m \cdot y)^{\mu_{x}}=\phi m^{\mu_{x}}$, i.e. $m^{\mu_{x}} \in M_{\phi}^{y^{\tau_{x}}}$. Therefore $\left(M_{\phi}^{y}\right)^{\mu_{x}} \subseteq M_{\phi}^{y^{\tau_{x}}}$. Similarly, $\left(M_{\phi}^{y^{\tau_{x}}}\right)^{\mu_{x}} \subseteq M_{\phi}^{y^{\tau_{x} \tau_{x}}}=M_{\phi}^{y}$ and thus $M_{\phi}^{y^{\tau_{x}}}=$ $\left(M_{\phi}^{y^{\tau x}}\right)^{\mu_{x}^{2}} \subseteq\left(M_{\phi}^{y}\right)^{\mu_{x}}$. We find that $\left(M_{\phi}^{y}\right)^{\mu_{x}}=M_{\phi}^{y^{\tau x}}$.

(iii) For $m \in M_{\phi}^{x}$, we have $m^{\mu_{x}}=\varepsilon m$ with $\varepsilon= \pm 1$ only depending on whether $\phi \in \Phi_{+}$ or $\phi \in \Phi_{-}$. By (ii), $m^{\mu_{y}} \in M_{\phi}^{x^{\tau_{y}}}$ and therefore $m^{\mu_{y} \mu_{x} \tau_{y}}=\varepsilon m^{\mu_{y}}$. By applying $\mu_{y}$,

$$
m^{\mu_{y} \mu_{x} \tau_{y} \mu_{y}}=\varepsilon m=m^{\mu_{x}}
$$

follows for all $m \in M_{\phi}^{x}$. Since $M=\bigoplus M_{\phi}^{x}$, by linearity we conclude that $\left(\mu_{x}\right)^{\mu_{y}}=$ $\mu_{x^{\tau} y}$.

Proof of theorem 3.9. By the definition of $\mathrm{U}(A, \Omega)$ and the fact that all $\mu_{e}$ have order at most 2, the theorem follows from Lemma 3.10|(iii)

\section{Matsuo algebras}

In Section 5, we will restrict our attention to the study of modules over Matsuo algebras, a special type of axial algebras. This section introduces these algebras and explains their connection with Fischer spaces and 3-transposition groups.

Definition 4.1. $\quad$ (i) A point-line geometry $\mathcal{G}$ is a pair $(\mathcal{P}, \mathcal{L})$ where $\mathcal{P}$ is a set whose elements are called points and $\mathcal{L}$ is a set of subsets of $\mathcal{P}$. The elements of $\mathcal{L}$ are called lines.

(ii) Two distinct points $x$ and $y$ of a point-line geometry are said to be collinear if there is a line containing both and we denote this by $x \sim y$. We write $x \nsim y$ if $x$ and $y$ are not collinear. If $x \nsim y$ for all $y \neq x$, then we call $x$ an isolated point.

(iii) A subspace of point-line geometry $(\mathcal{P}, \mathcal{L})$ is a point-line geometry $\left(\mathcal{P}^{\prime}, \mathcal{L}^{\prime}\right)$ such that:

- $\mathcal{P}^{\prime} \subseteq \mathcal{P}$ and $\mathcal{L} \subseteq \mathcal{L}^{\prime}$,

- if $x, y \in \mathcal{P}^{\prime}$ (with $x \neq y$ ) and $x, y \in \ell$ for some $\ell \in \mathcal{L}$, then $\ell \in \mathcal{L}^{\prime}$,

- if $\ell \in \mathcal{L}^{\prime}$, then all points of $\ell$ belong to $\mathcal{P}^{\prime}$.

Given a subset $X$ of $\mathcal{P}$, there is a unique minimal subspace of $(\mathcal{P}, \mathcal{L})$ containing the points of $X$; we call it the subspace generated by those points. 
(iv) Two points $x$ and $y$ of a point-line geometry are called connected if there exist points $x=x_{0}, x_{1}, \ldots, x_{n}=y$ such that $x_{i-1} \sim x_{i}$ for all $1 \leq i \leq n$. This relation defines an equivalence relation on the set of points of a point-line geometry. The subspaces generated by its equivalence classes are called the connected components of the point-line geometry.

(v) An isomorphism between two point-line geometries $\mathcal{G}=(\mathcal{P}, \mathcal{L})$ and $\mathcal{L}=\left(\mathcal{P}^{\prime}, \mathcal{L}^{\prime}\right)$ is a bijection $\theta: \mathcal{P} \rightarrow \mathcal{P}^{\prime}$ that induces a bijection between $\mathcal{L}$ and $\mathcal{L}^{\prime}$. We write $\mathcal{G} \cong \mathcal{G}^{\prime}$ and call $\mathcal{G}$ and $\mathcal{G}^{\prime}$ isomorphic if an isomorphism between them exists.

(vi) Let $\mathcal{G}$ be a point-line geometry such that through any two points there is at most one line and such that each line contains exactly three points. Such a point-line geometry is called a partial triple system. If $x$ and $y$ are collinear points in a partial triple system, there is a unique third point on the unique line through $x$ and $y$ and we will denote it by $x \wedge y$.

(vii) Let $\mathcal{G}$ be a partial triple system. We call $\mathcal{G}$ a Fischer space if each subspace generated by the points of two distinct intersecting lines is isomorphic to the dual affine plane of order 2 or the affine plane of order 3 . A sketch of these two point-line geometries is given in Figure 1 .
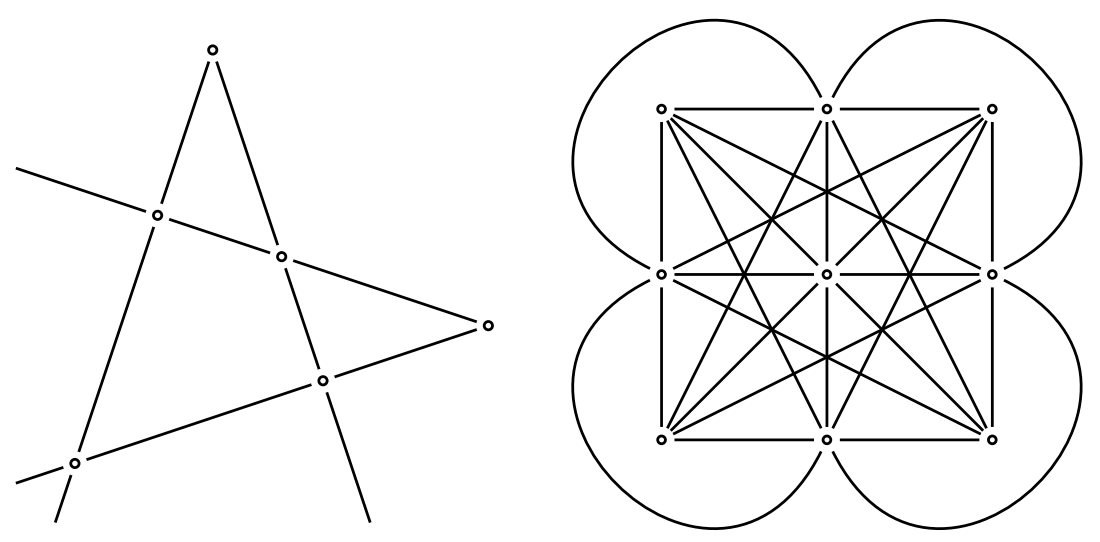

Figure 1: the dual affine plane of order 2 and the affine plane of order 3

The main motivation for studying Fischer spaces is their connection with 3-transposition groups, due to F. Buekenhout [Bue74]; see Proposition 4.5 below.

Definition 4.2 (See [Asc97]). A 3-transposition group is a pair $(G, D)$ where $D$ is a generating set of involutions of $G$ closed under conjugation such that the order of the product of any two elements of $D$ is at most 3 .

Example 4.3. (i) All symmetric groups together with their set of transpositions form a 3-transposition group.

(ii) The Fischer groups $F i_{22}, F i_{23}$ and $F i_{24}$ are 3 -transposition groups. 
In the connection between Fischer spaces and 3-transposition groups that we will need, it will be of importance to treat isolated points in Fischer spaces with some care.

Definition 4.4. (i) Let $(\mathcal{P}, \mathcal{L})$ be a Fischer space and let $\mathcal{P}^{\prime} \subseteq \mathcal{P}$ be the set of isolated points of $(\mathcal{P}, \mathcal{L})$. Then $\left(\mathcal{P} \backslash \mathcal{P}^{\prime}, \mathcal{L}\right)$ is clearly a Fischer space without isolated points that we will denote by $(\mathcal{P}, \mathcal{L})^{\circ}$.

(ii) If $\mathcal{G}=(\mathcal{P}, \mathcal{L})$ is a Fischer space, then we associate with each point $x \in \mathcal{P}$ an automorphism $\tau_{x} \in \operatorname{Aut}(\mathcal{G})$ defined as

$$
\tau_{x}: \mathcal{P} \rightarrow \mathcal{P}: y \mapsto \begin{cases}x \wedge y & \text { if } y \sim x, \\ y & \text { if } y \nsim x .\end{cases}
$$

Notice that $\tau_{x}$ is an involution unless $x$ is an isolated point (in which case $\tau_{x}$ is trivial). These involutions not only leave the set of points of the Fischer space invariant but also map collinear points to collinear points; hence they induce automorphisms of the Fischer space.

Now let $D=\left\{\tau_{x} \mid x \in \mathcal{P}\right.$ and $\left.\tau_{x} \neq 1\right\}$ and $G=\langle D\rangle \leq \operatorname{Aut}(\mathcal{G})$; then we define $f(\mathcal{G}):=(G, D)$.

(iii) Let $(G, D)$ be a 3 -transposition group. Then we write $(G, D)^{\circ}$ for the 3 -transposition group $(G / \mathrm{Z}(G),\{d \mathrm{Z}(G) \mid d \in D \backslash \mathrm{Z}(G)\})$.

(iv) Let $(G, D)$ be a 3 -transposition group. Let $\mathcal{P}=D$ and let $\mathcal{L}=\left\{\left\{c, d, c^{d}=d^{c}\right\} \mid\right.$ $\mathrm{o}(c d)=3\}$. Then $g(G, D):=(\mathcal{P}, \mathcal{L})$ is a point-line geometry.

Proposition 4.5 ([Bue74]). Let $\mathcal{G}$ be a Fischer space and let $(G, D)$ be a 3-transposition group. Then

(i) $g(G, D)$ is a Fischer space,

(ii) $f(\mathcal{G})$ is a 3-transposition group,

(iii) $f(g(G, D)) \cong(G, D)^{\circ}$,

(iv) $g(f(\mathcal{G})) \cong \mathcal{G}^{\circ}$.

Example 4.6. (i) The Fischer space corresponding to the 3-transposition group $S_{4}$ is the dual affine plane of order 2 .

(ii) The 3-transposition group related to the affine plane of order 3 is a semidirect product $3^{2}: 2$ where the action is given by inversion.

We now present the definition of Matsuo algebras, which will be instances of axial algebras; see Proposition 4.8 below. When the Matsuo algebra arises from a Fischer space, its fusion rules will be $\mathbb{Z} / 2 \mathbb{Z}$-graded; see Proposition 4.9 below.

Definition 4.7. Let $k$ be a field with $\operatorname{char}(k) \neq 2$, let $\alpha \in k \backslash\{0,1\}$ and let $\mathcal{G}=(\mathcal{P}, \mathcal{L})$ be a partial triple system. Define the Matsuo algebra $M_{\alpha}(\mathcal{G})$ as the $k$-vector space with 
basis $\mathcal{P}$ and multiplication defined by linearly extending

$$
x y= \begin{cases}x & \text { if } x=y, \\ 0 & \text { if } x \nsim y, \\ \frac{\alpha}{2}(x+y-x \wedge y) & \text { if } x \sim y,\end{cases}
$$

for all $x, y \in \mathcal{P}$.

The following proposition gives us a decomposition of a Matsuo algebra as a direct sum of eigenspaces for any $x \in \mathcal{P}$.

Proposition 4.8 ([HRS15a, Theorem 6.2]). For each $x \in \mathcal{P}$, the eigenspaces of $x$ in $M_{\alpha}(\mathcal{G})$ are

$$
\begin{aligned}
& \langle x\rangle, \text { its 1-eigenspace, } \\
& \langle y+x \wedge y-\alpha x \mid y \sim s\rangle \oplus\langle y \mid y \nsim x\rangle, \text { its 0-eigenspace, } \\
& \langle y-x \wedge y \mid y \sim x\rangle, \text { its } \alpha \text {-eigenspace, }
\end{aligned}
$$

and the algebra $M_{\alpha}(\mathcal{G})$ decomposes as a direct sum of these eigenspaces.

These decompositions satisfy the Jordan fusion rule $\Phi(\alpha)$ precisely when $\mathcal{G}$ is a Fischer space:

Proposition 4.9 ([HRS15a, Theorem 6.5]). Let $\mathcal{G}=(\mathcal{P}, \mathcal{L})$ be a partial triple system, with corresponding Matsuo algebra $A=M_{\alpha}(\mathcal{G})$. Then the Matsuo algebra $(A, \mathcal{P})$ is a $\Phi(\alpha)$-axial algebra if and only if $\mathcal{G}$ is a Fischer space.

Since the Jordan fusion rule $\Phi(\alpha)$ is $\mathbb{Z} / 2 \mathbb{Z}$-graded with $\Phi(\alpha)_{+}=\{1,0\}$ and $\Phi(\alpha)_{-}=$ $\{\alpha\}$, we can consider the Miyamoto involutions $\tau_{x} \in \operatorname{Aut}\left(M_{\alpha}(\mathcal{G})\right)$ for each $x \in \mathcal{P}$. Isolated points of the Fischer space should be treated with some care; they do not pose any serious difficulties, however, since they would give rise to trivial Miyamoto involutions. For simplicity, we nevertheless exclude this situation.

Proposition 4.10 ([HRS15a, Theorem 6.4]). Let $\mathcal{G}=(\mathcal{P}, \mathcal{L})$ be a Fischer space without isolated points, with corresponding Matsuo algebra $A=M_{\alpha}(\mathcal{G})$. Then:

(i) The pair $(A, \mathcal{P})$ is an axial representation of the 3-transposition group $f(\mathcal{G})$.

(ii) For each $x \in \mathcal{P}$, the Miyamoto involution $\tau_{x} \in \operatorname{Aut}(A)$ acts on $\mathcal{P}$ as the automorphism $\tau_{x}$ introduced in Definition 4.4(ii).

(iii) The axial representation $(A, \mathcal{P})$ is of unique type.

Proof. Statements (i) and (ii) follow from [HRS15a, Theorem 6.4]. We now show (iii). So let $D=\left\{\tau_{x} \mid x \in \mathcal{P}\right\} \subseteq \operatorname{Aut}(A)$; we have to show that the map $\tau: \mathcal{P} \rightarrow D: x \mapsto \tau_{x}$ is injective. Suppose $\tau_{x}=\tau_{y}$ for some $x, y \in \mathcal{P}$; then by (ii), $\tau_{x}$ and $\tau_{y}$ induce the same automorphism of the Fischer space $\mathcal{G}$. Since $\mathcal{G}$ has no isolated points, there exists some point $z \in \mathcal{P}$ such that $z \sim x$. Then $z^{\tau_{y}}=z^{\tau_{x}}=x \wedge z$ and therefore both $x$ and $y$ are the third point on the line through $z$ and $x \wedge z$. 
Remark 4.11. Let $\mathcal{G}=(\mathcal{P}, \mathcal{L})$ be a Fischer space without isolated points, with corresponding Matsuo algebra $A=M_{\alpha}(\mathcal{G})$. By Proposition 4.10|(ii), we can view the Miyamoto group $\operatorname{Miy}(A, \mathcal{P})=\left\langle\tau_{x} \mid x \in \mathcal{P}\right\rangle$ as a subgroup of either $\operatorname{Aut}(A)$ or $\operatorname{Aut}(\mathcal{G})$, whichever is the most convenient.

We now start from a 3-transposition group $(G, D)$ and we would like to construct an axial representation for $(G, D)$. Again, the situation giving rise to isolated points of the corresponding Fischer space should be treated with some care, and this works out nicely when we assume that $G$ is centerless.

Proposition 4.12. Let $(G, D)$ be a 3-transposition group with $Z(G)=1$. Then $(G, D)$ has an axial representation $\left(M_{\alpha}(\mathcal{G}), \mathcal{P}\right)$ for the Fischer space $\mathcal{G}=g(G, D)$.

Proof. Since $G$ is centerless, $(G, D)=(G, D)^{\circ}$. The statement now follows from Proposition 4.5(iii) together with Proposition 4.10|(i).

The following theorem, which is our main result in this section, shows that the group $\mathrm{U}\left(M_{\alpha}(\mathcal{G}), \mathcal{P}\right)$ can be interpreted as a universal 3-transposition group for the given Fischer space $\mathcal{G}$. Recall the definition of $\mathrm{U}(A)$ from Definition 3.5 above.

Theorem 4.13. Let $\mathcal{G}=(\mathcal{P}, \mathcal{L})$ be a Fischer space without isolated points. Consider the axial representation $\mathcal{A}=\left(M_{\alpha}(\mathcal{G}), \mathcal{P}\right)$ of the 3-transposition group $(G, D):=f(\mathcal{G})$. Then:

(i) $\left(\mathrm{U}(\mathcal{A}),\left\{t_{x} \mid x \in \mathcal{P}\right\}\right)$ is a 3-transposition group and $g\left(\mathrm{U}(\mathcal{A}),\left\{t_{x} \mid x \in \mathcal{P}\right\}\right) \cong \mathcal{G}$.

(ii) Let $\left(G^{\prime}, D^{\prime}\right)$ be a 3-transposition group such that $g\left(G^{\prime}, D^{\prime}\right) \cong \mathcal{G}$. Let $\varphi: \mathcal{P} \rightarrow D^{\prime}$ be an isomorphism between $\mathcal{G}$ and $g\left(G^{\prime}, D^{\prime}\right)$. The map defined by

$$
\theta: \mathrm{U}(\mathcal{A}) \rightarrow G^{\prime}: t_{x} \mapsto \varphi(x)
$$

is a group epimorphism and $G^{\prime} / \mathrm{Z}\left(G^{\prime}\right) \cong \mathrm{U}(\mathcal{A}) / \mathrm{Z}(\mathrm{U}(\mathcal{A})) \cong G$.

Proof. (i) Since all relations that hold in $\mathrm{U}(\mathcal{A})$ have even length and all $t_{x}$ have order at most 2 , all $t_{x}$ have order exactly 2 . Therefore $\left\{t_{x} \mid x \in \mathcal{P}\right\}$ is, by definition of $\mathrm{U}(\mathcal{A})$, a generating set of involutions invariant under conjugation. Since $\mathcal{G}$ has no isolated points, the axial representation $\mathcal{A}$ is of unique type and all $\tau_{x}$ are different. By Proposition 3.6[(i), all $t_{x}$ must also be different.

Let $x, y \in \mathcal{P}$; then, by Proposition 4.10, either $x=y$ or $x^{\tau_{y}}=x$ if $x \nsim y$ or $x^{\tau_{y}}=$ $x \wedge y$ if $x \sim y$. In the first case, $t_{x} t_{y}=1$. In the second case, $\left(t_{x} t_{y}\right)^{2}=t_{x} t_{x^{\tau}}=1$. In the third case, $y^{\tau_{x}}=x \wedge y$ and thus $\left(t_{x} t_{y}\right)^{3}=t_{x^{\tau} y} t_{y^{\tau_{x}}}=\left(t_{x \wedge y}\right)^{2}=1$. This proves that $\left(\mathrm{U}(\mathcal{A}),\left\{t_{x} \mid x \in \mathcal{P}\right\}\right)$ is indeed a 3 -transposition group.

Let $x, y, z \in \mathcal{P}$; then $t_{x}, t_{y}$ and $t_{z}$ lie on a line in $g\left(\mathrm{U}(\mathcal{A}),\left\{t_{x} \mid x \in \mathcal{P}\right\}\right)$ if and only if $\mathrm{o}\left(t_{x} t_{y}\right)=3$ and $\left(t_{x}\right)^{t_{y}}=t_{z}$. By our previous arguments, this happens if and only if $x \sim y$ and $z=x \wedge y$. 
(ii) For each $x \in \mathcal{P}, \varphi(x) \in D^{\prime}$ is an involution. Let $x, y \in \mathcal{P}$. If $x \sim y$, then, by definition of $g\left(G^{\prime}, D^{\prime}\right), \varphi(x)^{\varphi(y)}=\varphi(x \wedge y)=\varphi\left(x^{\tau_{y}}\right)$. If $x \nsim y$, then $\varphi(x) \varphi(y)$ has order 2 and $\varphi(x)^{\varphi(y)}=\varphi(x)=\varphi\left(x^{\tau_{y}}\right)$. This proves that $\theta$ is a group epimorphism. Consider the map $\theta^{\prime}: G^{\prime} \rightarrow G$ defined by $\varphi(x) \mapsto \tau_{x}$. Then $\operatorname{ker}\left(\theta^{\prime} \circ \theta\right)=\mathrm{Z}(\mathrm{U}(\mathcal{A}))$ by Proposition 3.7|(iii), Because $\theta$ is surjective, $\operatorname{ker}\left(\theta^{\prime}\right) \leq \mathrm{Z}\left(G^{\prime}\right)$ and, since $G$ is centerless, $\operatorname{ker}\left(\theta^{\prime}\right)=\mathrm{Z}\left(G^{\prime}\right)$. The isomorphism $G \cong \mathrm{U}(\mathcal{A}) / \mathrm{Z}(\mathrm{U}(\mathcal{A}))$ follows from Proposition 3.7|(iii) or by applying the previous argument to the 3 -transposition group $\left(G^{\prime}, D^{\prime}\right)=\left(\mathrm{U}(\mathcal{A}),\left\{t_{x} \mid x \in \mathcal{P}\right\}\right)$.

Example 4.14. Let $\mathcal{G}=(\mathcal{P}, \mathcal{L})$ be the affine plane of order 3. Then $\mathrm{U}\left(M_{\alpha}(\mathcal{G}), \mathcal{P}\right)$ is a semidirect product $3^{2}: S_{3}$ which is a central extension of the group $3^{2}: 2$ from Example 4.6(ii) by a cyclic group of order 3 .

\section{Modules over Matsuo algebras}

Theorem 3.9 tells us that we can get a group representation out of a module over an axial algebra. For Matsuo algebras, the converse will also be true. In Theorem 5.1 we construct a module over a Matsuo algebra from such a group representation.

Theorem 5.1. Let $\mathcal{G}=(\mathcal{P}, \mathcal{L})$ be a Fischer space. Let $k$ be a field with $\operatorname{char}(k) \neq 2$ and $\alpha \in k \backslash\{0,1\}$. Consider the Matsuo algebra $M_{\alpha}(\mathcal{G})$. Let $V$ be a $k$-vector space and $\rho: \mathrm{U}\left(M_{\alpha}(\mathcal{G}), \mathcal{P}\right) \rightarrow \mathrm{GL}(V)$ a group homomorphism. Since $\rho\left(t_{x}\right)^{2}=1$ for every $x \in \mathcal{P}$ and $\operatorname{char}(k) \neq 2$, we can decompose $V$ as a direct sum of the 1-and (-1)-eigenspace of $\rho\left(t_{x}\right)$. The action defined by linearly extending

$$
v \cdot x= \begin{cases}0 & \text { if } v^{\rho\left(t_{x}\right)}=v \\ \alpha v & \text { if } v^{\rho\left(t_{x}\right)}=-v\end{cases}
$$

for each $x \in \mathcal{P}$, equips $V$ with the structure of an $M_{\alpha}(\mathcal{G})$-module.

We start by mimicking Lemma 3.10(i) with $\mu_{x}$ replaced by $\rho\left(t_{x}\right)$.

Lemma 5.2. For every $v \in V, a \in M_{\alpha}(\mathcal{G})$ and $x \in \mathcal{P},(v \cdot a)^{\rho\left(t_{x}\right)}=v^{\rho\left(t_{x}\right)} \cdot a^{\tau_{x}}$.

Proof. Since $M_{\alpha}(\mathcal{G})$ is spanned by the elements of $\mathcal{P}$ and the action of $M_{\alpha}(\mathcal{G})$ is linear by definition, we may assume that $a=y \in \mathcal{P}$. Since $V$ is decomposable into a 1 - and $(-1)$-eigenspace of $\rho\left(t_{x}\right)$, it suffices to consider the cases where $v$ is a 1- or $(-1)$-eigenvector of $\rho\left(t_{x}\right)$. If $v^{\rho\left(t_{y}\right)}=v$ then $v \cdot y=0$ and $(v \cdot y)^{\rho\left(t_{x}\right)}=0$. Moreover

$$
\left(v^{\rho\left(t_{x}\right)}\right)^{\rho\left(t_{y} \tau_{x}\right)}=v^{\rho\left(t_{x}\right) \rho\left(t_{x} t_{y} t_{x}\right)}=v^{\rho\left(t_{x}\right)}
$$

and hence $v^{\rho\left(t_{x}\right)} \cdot y^{\tau_{x}}=0$. In the second case, $v^{\rho\left(t_{y}\right)}=-v$ and therefore $v \cdot y=\alpha v$ and $(v \cdot y)^{\rho\left(t_{x}\right)}=\alpha v^{\rho\left(t_{x}\right)}$. Now

$$
\left(v^{\rho\left(t_{x}\right)}\right)^{\rho\left(t_{y} \tau_{x}\right)}=v^{\rho\left(t_{x}\right) \rho\left(t_{x} t_{y} t_{x}\right)}=-v^{\rho\left(t_{x}\right)}
$$

and thus $v^{\rho\left(t_{x}\right)} \cdot y^{\tau_{x}}=\alpha v^{\rho\left(t_{x}\right)}$. In both cases, $(v \cdot y)^{\rho\left(t_{x}\right)}=v^{\rho\left(t_{x}\right)} \cdot y^{\tau_{x}}$. 
Proof of Theorem 5.1. Let $x \in \mathcal{P}$. The vector space $V$ decomposes into a 1- and $(-1)$-eigenspace of $\rho\left(t_{x}\right)$. By definition of the action of $x, V$ decomposes as $V=V_{0}^{x} \oplus V_{\alpha}^{x}$ where $V_{0}^{x}$ and $V_{\alpha}^{x}$ are the 1- and (-1)-eigenspace of $\rho\left(t_{x}\right)$, respectively. It only remains to verify that the fusion rule is satisfied. Let $A=M_{\alpha}(\mathcal{G})$. For $v \in V_{0}^{x}$ and $a \in A_{\{1,0\}}^{x}$ (resp. $v \in V_{\alpha}^{x}$ and $\left.a \in A_{\alpha}^{x}\right)$ we have $v^{\rho\left(t_{x}\right)}=v\left(\right.$ resp. $\left.v^{\rho\left(t_{x}\right)}=-v\right)$ and $a^{\tau_{x}}=a\left(\right.$ resp. $\left.a^{\tau_{x}}=-a\right)$. By Lemma 5.2 .

$$
(v \cdot a)^{\rho\left(t_{x}\right)}=v^{\rho\left(t_{x}\right)} \cdot a^{\tau_{x}}=v \cdot a
$$

and thus $v \cdot a$ is a 1-eigenvector of $\rho\left(t_{x}\right)$. Therefore $v \cdot a$ belongs to $V_{0}^{x}$. If $v \in V_{0}^{x}$ and $a \in A_{\alpha}^{x}$ (resp. $v \in V_{\alpha}^{x}$ and $\left.a \in A_{\{1,0\}}^{x}\right)$, then $v^{\rho\left(t_{x}\right)}=v\left(\right.$ resp. $\left.v^{\rho\left(t_{x}\right)}=-v\right)$ and $a^{\tau_{x}}=-a$ (resp. $a^{\tau_{x}}=a$ ). In both cases, by Lemma 5.2 .

$$
(v \cdot a)^{\rho\left(t_{x}\right)}=v^{\rho\left(t_{x}\right)} \cdot a^{\tau_{x}}=-v \cdot a .
$$

Therefore $v \cdot a$ is a 1-eigenvector of $\rho\left(t_{x}\right)$ and hence $v \cdot a \in V_{\alpha}^{x}$.

Theorem 5.1 has the important consequence that for a Matsuo algebra $A$, the study of representations for the group $\mathrm{U}(A)$ is equivalent to the study of certain $A$-modules, as we now point out. Recall from Definition 3.1 that $\mathbf{M o d}_{A}$ is the category of all $A$-modules, for any axial algebra $A$.

Corollary 5.3. Let $\mathcal{G}=(\mathcal{P}, \mathcal{L})$ be a Fischer space, let $A=\left(M_{\alpha}(\mathcal{G}), \mathcal{P}\right)$ be its Matsuo algebra and let $\mathrm{U}(A)$ be the universal group introduced in Definition 3.5. Let $\mathbf{M o d}_{A}^{(1)}$ be the full subcategory of $\mathbf{M o d}_{A}$ of $A$-modules $M$ for which $M_{1}^{x}=\{0\}$ for all $x \in \mathcal{P}$. Then the category $\mathbf{M o d}_{A}^{(1)}$ is equivalent to the category $\operatorname{Rep}_{\mathrm{U}(A)}$ of $\mathrm{U}(A)$-representations.

Proof. Using Theorem 5.1, we can associate to each linear representation $\rho$ of $\mathrm{U}(A)$ an $A$-module $M_{\rho}$ with $\left(M_{\rho}\right)_{1}^{x}=\{0\}$ for all $x$. We first observe that the resulting map $F: \rho \rightsquigarrow M_{\rho}$ is a functor. Indeed, if $\theta: \rho \rightarrow \rho^{\prime}$ is a homomorphism of $\mathrm{U}(A)$-representations, then for each $x \in \mathcal{P}, \theta$ maps $\rho\left(t_{x}\right)$-eigenspaces to $\rho^{\prime}\left(t_{x}\right)$-eigenspaces, hence by Theorem 5.1 again, $\theta$ induces a homomorphism of the corresponding $A$-modules. It is now also clear that for given $\mathrm{U}(A)$-representations $\rho, \rho^{\prime}$, the functor $F$ induces a bijection between the morphisms from $\rho$ to $\rho^{\prime}$ and the morphisms from $M_{\rho}$ to $M_{\rho}^{\prime}$; hence $F$ is a fully faithful functor.

To show that the functor $F$ is essentially surjective, let $M$ be an arbitrary $A$-module with $M_{1}^{x}=\{0\}$ for all $x \in \mathcal{P}$. By Theorem 3.9, there is an associated linear representation $\rho$ of the group $\mathrm{U}(A)$; we claim that $M_{\rho} \cong M$. Indeed, since $\rho\left(t_{x}\right)=\mu_{x}$ and $M_{1}^{x}=\{0\}$, we see that $x \cdot m=0(\operatorname{resp} . x \cdot m=\alpha m)$ if and only if $m^{\rho\left(t_{x}\right)}=m\left(\right.$ resp. $\left.m^{\rho\left(t_{x}\right)}=-m\right)$ for all $m \in M$ and $x \in \mathcal{P}$. By definition of $M_{\rho}$, this proves the claim.

This shows that the functor $F$ is an equivalence.

More generally, given any module $M$ over the Matsuo algebra $M_{\alpha}(\mathcal{G})$, Theorem 3.9 gives us a representation $\rho$ of the group $\mathrm{U}\left(M_{\alpha}(\mathcal{G}), \mathcal{P}\right)$. The module constructed by Theorem 5.1 out of $\rho$ resembles $M$, with the important difference that all 1-eigenvectors of an axis $x \in \mathcal{P}$ have become 0 -eigenvectors. (The action on the 0 - and $\alpha$-eigenvectors 
remains unchanged.) This leaves of course the question what the role of a 1-eigenvector is inside a module. Since a regular module, i.e., the axial algebra as a module over itself, contains non-trivial 1-eigenspaces, we definitely want to allow the existence of 1-eigenspaces in Definition 3.1. We will now prove that, under certain conditions, this is the only way a 1-eigenspace can turn up in a module over a Matsuo algebra.

From now on, we would like to restrict to Matsuo algebras over connected Fischer spaces without isolated points. This is not a serious restriction, as the following proposition allows to generalize results to arbitrary Fischer spaces without isolated points by looking at their connected components.

Proposition 5.4 ([HRS15a, Theorem 6.2]). Let $\mathcal{G}$ be a Fischer space and let $\left\{\mathcal{G}_{i} \mid i \in I\right\}$ be the set of its connected components. Then $M_{\alpha}\left(\mathcal{G}_{i}\right) \cong \bigoplus_{i \in I} M_{\alpha}\left(\mathcal{G}_{i}\right)$.

The connectedness of a Fischer space has the following implications on its Matsuo algebra.

Lemma 5.5. Let $\mathcal{G}=(\mathcal{P}, \mathcal{L})$ be a Fischer space and let $M_{\alpha}(\mathcal{G})$ be its Matsuo algebra. Let $D=\left\{\tau_{x} \mid x \in \mathcal{P}\right\}$ and $G=\langle D\rangle \leq \operatorname{Aut}\left(M_{\alpha}(\mathcal{G})\right)$. The following statements are equivalent.

(a) The Fischer space $\mathcal{G}$ is connected.

(b) The action of $G$ on $\mathcal{P}$ is transitive.

(c) The set $D$ of Miyamoto involutions is a conjugacy class of $G$.

(d) The set $\left\{t_{x} \mid x \in \mathcal{P}\right\}$ is a conjugacy class of $\mathrm{U}\left(M_{\alpha}(\mathcal{G}), \mathcal{P}\right)$.

Proof. Suppose the Fischer space $\mathcal{G}$ is connected. Let $x, y \in \mathcal{P}$. Then there exists a path $x, x_{1}, \ldots, x_{n}, y$ from $x$ to $y$. Now

$$
x^{\tau_{x \wedge x_{1}} \tau_{x_{1} \wedge x_{2} \cdots \tau_{x_{n} \wedge y}}}=y
$$

and therefore $G$ acts transitively on $\mathcal{P}$. Conversely, by Proposition 4.10, all Miyamoto involutions stabilize the connected components of the Fischer space. Therefore, the Fischer space is connected when $G$ acts transitively on $\mathcal{P}$. The equivalence between (b) and (c) follows from Proposition 2.9. By definition of the group $\mathrm{U}\left(M_{\alpha}(\mathcal{G}), \mathcal{P}\right)$, it follows that $(\mathrm{c})$ and $(\mathrm{d})$ are equivalent.

From now on, let $\mathcal{G}=(\mathcal{P}, \mathcal{L})$ be a connected Fischer space without isolated points. Consider the axial representation $\left(M_{\alpha}(\mathcal{G}), \mathcal{P}\right)$ of the 3-transposition group $(G, D):=f(\mathcal{G})$. Let $M$ be an $M_{\alpha}(\mathcal{G})$-module and suppose $M_{1}^{x} \neq\{0\}$ for some (and hence every) axis $x \in \mathcal{P}$. We will prove in Theorem 5.8 that, under certain conditions, $M$ contains a submodule that is a quotient of $M_{\alpha}(\mathcal{G})$ as an $M_{\alpha}(\mathcal{G})$-module. First, we will construct the submodule. We introduce some notation.

Definition 5.6. Let $M$ be an $M_{\alpha}(\mathcal{G})$-module and suppose $M_{1}^{x} \neq\{0\}$ for each $x \in \mathcal{P}$. 
(i) Let

$$
U:=\left\langle\mu_{x} \mid x \in \mathcal{P}\right\rangle \leq \mathrm{GL}(M) .
$$

Denote the group $\mathrm{U}\left(M_{\alpha}(\mathcal{G}), \mathcal{P}\right)$ by $T$ and consider, as in Proposition 3.6](i) and Theorem 3.9, the group epimorphisms defined by

$$
\begin{aligned}
& \mu: T \rightarrow U: t_{x} \mapsto \mu_{x}, \\
& \tau: T \rightarrow G: t_{x} \mapsto \tau_{x} .
\end{aligned}
$$

(ii) For each $x \in \mathcal{P}$, we define a subgroup $U_{x}=\mu\left(C_{T}\left(t_{x}\right)\right)$ of $U$, where $C_{T}\left(t_{x}\right)$ is the centraliser of $t_{x}$ in $T$.

(iii) Fix $x \in \mathcal{P}$. Let $m \in M_{1}^{x}$ with $m \neq 0$. Let

$$
m_{x}=\sum_{\mu \in U_{x}} m^{\mu} .
$$

Because we assume that $\mathcal{G}$ is connected, the group $T$ acts transitively on the set $\left\{t_{x} \mid x \in \mathcal{P}\right\}$. Thus for every $y \in \mathcal{P}$ there exists $t \in T$ such that $\left(t_{x}\right)^{t}=t_{y}$ and we define

$$
m_{y}=\left(m_{x}\right)^{\mu(t)} .
$$

By Lemma 5.7(iv) below, this definition of $m_{y}$ is independent of the choice of $t$.

Lemma 5.7. Let $y \in \mathcal{P}, t \in T$ and $\phi \in\{1,0, \alpha\}$. Suppose $t^{\prime} \in T$ such that $\left(t_{x}\right)^{t}=\left(t_{x}\right)^{t^{\prime}}$. Then the following hold:

(i) $\left(t_{y}\right)^{t}=t_{y^{\tau(t)}}$,

(ii) $\left(M_{\phi}^{y}\right)^{\mu(t)}=M_{\phi}^{y^{\tau(t)}}$,

(iii) $U_{x} \mu(t)=U_{x} \mu\left(t^{\prime}\right)$,

(iv) $\left(m_{x}\right)^{\mu(t)}=\left(m_{x}\right)^{\mu\left(t^{\prime}\right)}$,

(v) $m_{y} \in M_{1}^{y}$,

(vi) $\left(m_{y}\right)^{\mu(t)}=m_{y^{\tau(t)}}$.

Proof. (i) This follows by definition of $\mathrm{U}\left(M_{\alpha}(\mathcal{G}), \mathcal{P}\right)$.

(ii) By Lemma 3.10|(ii), this follows because $\mu$ and $\tau$ are group homomorphisms.

(iii) Since $C_{\mathrm{U}\left(M_{\alpha}(\mathcal{G}), \mathcal{P}\right)}\left(t_{x}\right) t=C_{\mathrm{U}\left(M_{\alpha}(\mathcal{G}), \mathcal{P}\right)}\left(t_{x}\right) t^{\prime}$ whenever $\left(t_{x}\right)^{t}=\left(t_{x}\right)^{t^{\prime}}$, also $U_{x} \mu(t)=$ $U_{x} \mu\left(t^{\prime}\right)$.

(iv) By (iii),

$$
\left(m_{x}\right)^{\mu(t)}=\sum_{\mu \in U_{x} \mu(t)} m^{\mu}=\sum_{\mu \in U_{x} \mu\left(t^{\prime}\right)} m^{\mu}=\left(m_{x}\right)^{\mu\left(t^{\prime}\right)} .
$$


(v) Let $t \in C_{\mathrm{U}\left(M_{\alpha}(\mathcal{G}), \mathcal{P}\right)}\left(t_{x}\right)$. Since $t_{x}=\left(t_{x}\right)^{t}=t_{x^{\tau(t)}}$, it follows from Proposition 4.10) (iii) that $x^{\tau(t)}=x$. Therefore $m^{\mu(t)} \in\left(M_{1}^{x}\right)^{\mu(t)}=M_{1}^{x^{\tau(t)}}=M_{1}^{x}$. Thus $m_{x}=$ $\sum_{\mu \in U_{x}} m^{\mu} \in M_{1}^{x}$. For $y \in \mathcal{P}$, let $t \in \mathrm{U}\left(M_{\alpha}(\mathcal{G}), \mathcal{P}\right)$ such that $\left(t_{x}\right)^{t}=t_{x^{\tau(t)}}=t_{y}$ and thus $x^{\tau(t)}=y$. Now, $m_{y}=m_{x}^{\mu(t)} \in\left(M_{1}^{x}\right)^{\mu(t)}=M_{1}^{x^{\tau(t)}}=M_{1}^{y}$.

(vi) Because $\left(t_{x}\right)^{t^{\prime}}=t_{y}, x^{\tau\left(t^{\prime}\right)}=y$. Since $\left(t_{x}\right)^{t^{\prime} t}=t_{x^{\tau\left(t^{\prime} t\right)}}=t_{y^{\tau(t)}}$, it follows, by $(\star)$, that $m_{y^{\tau(t)}}=\left(m_{x}\right)^{\mu\left(t^{\prime} t\right)}=\left(m_{y}\right)^{\mu(t)}$.

Theorem 5.8. Consider the setting of Definition 5.6. The subspace $\left\langle m_{y} \mid y \in \mathcal{P}\right\rangle$ is a submodule of $M$ and the map, defined by

$$
M_{\alpha}(\mathcal{G}) \rightarrow\left\langle m_{y} \mid y \in \mathcal{P}\right\rangle: y \mapsto m_{y},
$$

is a surjective homomorphism of $M_{\alpha}(\mathcal{G})$-modules. In particular, $\left\langle m_{y} \mid y \in \mathcal{P}\right\rangle$ is a quotient of the regular module for $M_{\alpha}(\mathcal{G})$.

Proof. It suffices to prove that for idempotents $y, z \in \mathcal{P}$

$$
\begin{array}{ll}
m_{z} \cdot z=m_{z} ; & \text { if } y \nsim z ; \\
m_{y} \cdot z=0 & \text { if } y \sim z ; \\
\left(m_{y}+m_{y \wedge z}-\alpha m_{z}\right) \cdot z=0 & \\
\left(m_{y}-m_{y \wedge z}\right) \cdot z=\alpha\left(m_{y}-m_{y \wedge z}\right) & \text { if } y \sim z .
\end{array}
$$

Statement (1) follows from Lemma 5.7)(v). For (2), let $z, y \in \mathcal{P}$ and $y \nsim z$. This implies that $z \in\left(M_{\alpha}(\mathcal{G})\right)_{0}^{y}$. By Lemma [5.7](v), $m_{y} \in M_{1}^{y}$. From the fusion rule $M_{1}^{y} \cdot\left(M_{\alpha}(\mathcal{G})\right)_{0}^{y} \subseteq\{0\}$ we infer $m_{y} \cdot z=0$. For (3) and (4), let $z, y \in \mathcal{P}$ and $y \sim z$. Since $\left(m_{y}-m_{y \wedge z}\right)^{\mu_{z}}=m_{y \wedge z}-m_{y}$ by Lemma 5.7)(vi), (4) follows. For (3), note that

$$
m_{z} \cdot(y+z \wedge y-\alpha z)=0
$$

since $m_{z} \in M_{1}^{z}, y+z \wedge y-\alpha z \in\left(M_{\alpha}(\mathcal{G})\right)_{0}^{z}$ and $M_{1}^{z} \cdot\left(M_{\alpha}(\mathcal{G})\right)_{0}^{z} \subseteq\{0\}$. Interchanging the roles of $y, z$ and $y \wedge z$ in this relation and relation (4) gives us the following 5 relations:

$$
\begin{aligned}
& m_{y} \cdot(z+y \wedge z-\alpha y)=0, \\
& m_{z} \cdot(y \wedge z+y-\alpha z)=0, \\
& m_{y \wedge z} \cdot(y+z-\alpha y \wedge z)=0 \\
& \left(m_{z}-m_{y \wedge z}\right) \cdot y-\alpha\left(m_{z}-m_{y \wedge z}=0,\right. \\
& \left(m_{y}-m_{z}\right) \cdot y \wedge z-\alpha\left(m_{y}-m_{z}\right)=0 .
\end{aligned}
$$

It is easy to verify, using relation (1), that $(5)-(6)+(7)+(8)-(9)$ gives us exactly (3).

Remark 5.9. We do not know whether it is always possible to choose $m \in M_{1}^{x}$ such that $m_{x} \neq 0$. 
Theorem 5.11 will tell us when the map from Theorem 5.8 is an isomorphism. The proof makes use of a Frobenius form for Matsuo algebras defined in Lemma 5.10 below. The only obstructions are the facts that $m_{x}$ might be zero or that the Frobenius form might be degenerate.

Lemma 5.10 ([HRS15b, Corollary 7.4]). Let $\mathcal{G}$ be a Fischer space. The Matsuo algebra $M_{\alpha}(\mathcal{G})$ is a Frobenius axial algebra. When $\mathcal{G}$ is connected, its Frobenius form is, up to scalar, uniquely determined. It is given by

$$
\langle x, y\rangle= \begin{cases}1 & \text { if } x=y, \\ \frac{\alpha}{2} & \text { if } x \sim y, \\ 0 & \text { if } x \nsim y,\end{cases}
$$

for all $x, y \in \mathcal{P}$.

Theorem 5.11. Let $\mathcal{G}=(\mathcal{P}, \mathcal{L})$ be a connected Fischer space without isolated points. Let $M$ be an $M_{\alpha}(\mathcal{G})$-module, $x \in \mathcal{P}, m \in M_{1}^{x} \backslash\{0\}$ and define

$$
m_{x}=\sum_{\mu \in U_{x}} m^{\mu}
$$

Let $U=\left\langle\mu_{x} \mid x \in \mathcal{P}\right\rangle \leq \mathrm{GL}(M)$. If $m_{x} \neq 0$ and the Frobenius form of $M_{\alpha}(\mathcal{G})$ is nondegenerate, then $\left\langle\left(m_{x}\right)^{\mu} \mid \mu \in U\right\rangle$ is a regular submodule of $M_{\alpha}(\mathcal{G})$, i.e., it is isomorphic to $M_{\alpha}(\mathcal{G})$ as $M_{\alpha}(\mathcal{G})$-module.

Proof. It suffices to prove that the map from Theorem 5.8 is injective. Let $m_{a}$ be the image of $a \in M_{\alpha}(\mathcal{G})$ under this map. We shall prove that for the stated conditions, $m_{a} \neq 0$ when $a \neq 0$. Since we require $\mathcal{G}$ to be connected, for every $y \in \mathcal{P}$, there exists a $\mu \in U$ such that $m_{y}=\left(m_{x}\right)^{\mu}$. Because $m_{x} \neq 0$ and $\mu \in \mathrm{GL}(M), m_{y} \neq 0$ for all $y \in \mathcal{P}$.

Suppose that $a \in M_{\alpha}(\mathcal{G})$ and $m_{a}=0$. Let $y \in \mathcal{P}$ be an arbitrary axis and write $a=a_{1}+a_{0}+a_{\alpha}$ where $a_{\phi} \in\left(M_{\alpha}(\mathcal{G})\right)_{\phi}^{y}$. Then

$$
m_{a}=m_{a_{1}}+m_{a_{0}}+m_{a_{\alpha}}=0 .
$$

Since $0=m_{a} \cdot y=m_{a \cdot y}$

$$
m_{a_{1}}+\alpha m_{a_{\alpha}}=0
$$

We also have $0=m_{a}^{\mu_{y}}=m_{a^{\tau} y}$ and therefore

$$
m_{a_{1}}+m_{a_{0}}-m_{a_{\alpha}}=0 .
$$

From these three equations it follows that $m_{a_{1}}=m_{a_{0}}=m_{a_{\alpha}}=0$.

Since the 1-eigenspace of $y$ in $M_{\alpha}(\mathcal{G})$ is spanned by $y, a_{1}=\lambda y$ for some $\lambda \in k$. Because $m_{y} \neq 0$, we conclude that $a_{1}$ must be zero. As our choice of $y \in \mathcal{P}$ was arbitrary, we infer that for every axis in $\mathcal{P}$ the component of $a$ in its 1-eigenspace is zero.

Let $\langle$,$\rangle be the Frobenius form for M_{\alpha}(\mathcal{G})$ as given by Lemma 5.10. Since eigenvectors corresponding to different eigenvalues are perpendicular to each other, our previous conclusion is equivalent to

$$
\langle a, y\rangle=0 \text { for all } y \in \mathcal{P} .
$$


The elements of $\mathcal{P}$ span $M_{\alpha}(\mathcal{G})$ and therefore $\langle a, n\rangle=0$ for all $n \in M_{\alpha}(\mathcal{G})$. Because the bilinear form $\langle$,$\rangle is non-degenerate, a=0$.

Remark 5.12. (i) Let $\mathcal{G}$ be a connected Fischer space without isolated points and let $M$ be a finite-dimensional Frobenius $M_{\alpha}(\mathcal{G})$-module. Suppose that the Frobenius form of $M_{\alpha}(\mathcal{G})$ is non-degenerate. Combining Theorem 5.11, Lemma 5.10 and Proposition 3.4, we can decompose $M$ as a direct sum of regular modules and a module $M^{\prime}$ for which $\sum_{\mu \in U_{x}} m^{\mu}=0$ for every $m \in\left(M^{\prime}\right)_{1}^{x}$ and every $x \in \mathcal{P}$.

(ii) Suppose $\mathcal{G}$ is a finite connected Fischer space without isolated points. Let $A$ be its collinearity matrix. The condition that the Frobenius form from Lemma 5.10 is non-degenerate can be expressed as $\operatorname{det}\left(I+\frac{\alpha}{2} A\right) \neq 0$. From this, it is clear that this can only fail for a finite number of choices for $\alpha$.

(iii) Since the Miyamoto group of $M_{\alpha}(\mathcal{G})$ acts transitively on the points of a connected Fischer space $\mathcal{G}$, the number of lines through a point is a constant $d \in \mathbb{N}$ if $\mathcal{G}$ is finite. The number of points collinear with any given point is then $2 d$. The vector of all ones is therefore a $(1+\alpha d)$-eigenvector of $I+\frac{\alpha}{2} A$ where $A$ is the collinearity matrix of $\mathcal{G}$. It is easy to verify that

$$
\left(\sum_{x \in \mathcal{P}} x\right) a=(1+\alpha d) a
$$

for all $a \in M_{\alpha}(\mathcal{G})$. If $1+\alpha d=0$ and hence $\operatorname{det}\left(I+\frac{\alpha}{2} A\right)=0$, then $\left\langle\sum_{x \in \mathcal{P}} x\right\rangle$ is a 1-dimensional $M_{\alpha}(\mathcal{G})$-submodule of $M_{\alpha}(\mathcal{G})$. The quotient module is then an $M_{\alpha}(\mathcal{G})$-module non-isomorphic to $M_{\alpha}(\mathcal{G})$ with a non-trivial 1-eigenspace for every axis $x \in \mathcal{P}$. The condition that the Frobenius form must be non-degenerate can therefore not be omitted. Note that if $1+\alpha d \neq 0$, then $M_{\alpha}(\mathcal{G})$ is a unital algebra with unit $(1+\alpha d)^{-1}\left(\sum_{x \in \mathcal{P}} x\right)$.

\section{References}

[Asc97] M. Aschbacher. 3-Transposition groups, volume 124 of Cambridge tracts in mathematics. Cambridge University Press, 1997.

[Bor86] Richard E. Borcherds. Vertex algebras, Kac-Moody algebras, and the Monster. Proc. Natl. Acad. Sci. USA, 83:3068-3071, 1986.

[Bue74] Francis Buekenhout. La géometrie des groupes de Fischer. Unpublished notes, 1974.

[FLM88] Igor Frenkel, James Lepowsky, and Arne Meurman. Vertex Operator Algebras and the Monster, volume 134 of Pure and applied mathematics. Academic Press, Inc., 1988.

[Gri82] Robert L. Griess. The friendly giant. Invent. math., 69:1-102, 1982.

[Hal06] Jonathan I. Hall. A characterization of the full wreath product. J. Algebra, 300(2):529$554,2006$.

[HRS15a] Jonathan I. Hall, Felix Rehren, and Sergey Shpectorov. Primitive axial algebras of jordan type. J. Algebra, 437:79-115, 2015. 
[HRS15b] Jonathan I. Hall, Felix Rehren, and Sergey Shpectorov. Universal axial algebras and a theorem of Sakuma. J. Algebra, 421:394-424, 2015.

[HSS17] Jonathan I. Hall, Yoav Segev, and Sergey Shpectorov. Miyamoto involutions in axial algebras of Jordan type half. Israel Journal of Mathematics, 2017.

[IPS10] Alexander A. Ivanov, Dmitrii V. Pasechnik, and Ákos Seress. Majorana representations of the symmetric group of degree 4. J. Algebra, 324:2432-2463, 2010.

[Iva09] Alexander A. Ivanov. The Monster Group and Majorana Involutions, volume 176 of Cambridge tracts in mathematics. Cambridge University Press, 2009.

[Jac68] Nathan Jacobson. Structure and Representations of Jordan Algebras, volume 39 of Colloquium Publications. American Mathematical Society, 1968.

[Reh15] Felix G. Rehren. Axial algebras. PhD thesis, University of Birmingham, 2015.

[Tit84] Jacques Tits. On R. Griess' "Friendly giant". Invent. math., 78:491-499, 1984. 